\title{
Investigation of Recurrent Cases of Head and Neck Cancer after Initial Treatment
}

\author{
Ryosuke Kitoh $^{1}$ Kentaro Mori ${ }^{1}$ Shin-ichi Usami ${ }^{1}$ \\ ${ }^{1}$ Department of Otorhinolaryngology, Shinshu University School of \\ Medicine, Matsumoto, Nagano, Japan \\ Int J Pract Otolaryngol 2019;2:e11-e17.
}

\author{
Address for correspondence Ryosuke Kitoh, MD, PhD, Department of \\ Otorhinolaryngology, Shinshu University School of Medicine, 3-1-1 \\ Asahi, Matsumoto, Nagano 390-8621, Japan \\ (e-mail: ryosuke@shinshu-u.ac.jp).
}

\begin{abstract}
Keywords

- recurrence

- head and neck cancer

- follow-up

- salvage

The optimal follow-up strategy for patients with head and neck cancer after primary treatment is still under debate. This study aimed to review the data of patients with recurrence and evaluate our follow-up strategy. Patients with head and neck cancer who underwent surgery or chemoradiotherapy as the primary treatment between 2012 and 2016 were enrolled. All the patients were carefully followed up at our institution via intensive clinical examination and routine computed tomography (CT) and positron emission tomography (PET)/CT. Recurrence was detected in 20 of the patients treated by surgery and 25 of 96 patients treated by chemoradiotherapy. In more than $90 \%$ of the patients, recurrence occurred within 2 years of the primary treatment. Among the patients with recurrence, 11 showed local recurrence, 17 showed regional recurrence, and 17 others showed distant metastasis. In almost all patients with distant metastases, recurrence was detected using CT or PET/CT, whereas among the patients with local or regional recurrence, recurrence was detected based on the patients' complaints and their clinical examination results. Only salvage surgery was observed to be associated with disease-free survival after recurrence. Thus, analysis of the recurrence patterns and appropriate surveillance can result in improved salvage treatment for patients with recurrence.
\end{abstract}

\section{Introduction}

The Head and Neck Cancer Guidelines were revised in 2018. This revised edition includes an additional statement regarding posttreatment follow-up. Here, we report on the review of conventional follow-up observation after initial treatment at our hospital based on recurrent cases and investigation on future follow-up observation policies.

\section{Materials and Methods}

The study included patients who underwent surgery or chemoradiotherapy (CRT) as initial treatment during the 5-year period from 2012 to 2016 at the Department of Otorhinolaryngology, Shinshu University School of Medicine, Matsumoto, Nagano, Japan.

received

January 18, 2019

accepted

March 27, 2019
DOI https://doi.org/ 10.1055/s-0039-1692466. eISSN 2569-1783.
As follow-up observation, patients were examined once per month for the first 2 years and every 2-3 months thereafter. Those who were treated with chemoradiotherapy (CRT) underwent computed tomography (CT) (or magnetic resonance imaging [MRI]) in the first 2 months and positron emission tomography (PET) in the first 3 months. For patients who underwent surgery, baseline images were obtained within 6 months after surgery. In all patients, imaging examinations were performed every 6 months until 2 years after the treatment and every 6 to 12 months from the third year onward. Blood sampling and chest X-rays were also performed once every 6 months.

The diagnosis of recurrence was defined as recurrence being confirmed by pathological diagnosis or imaging. The duration until recurrence was considered to start from the
Copyright (c) 2019 Georg Thieme Verlag License terms KG Stuttgart · New York 
e12 Recurrence after Head and Neck Cancer Treatment Kitoh et al.

treatment start date. For statistical analyses, Mann-Whitney test, Fisher's exact test, and the log-rank test were performed using PASW statistics version 18 (SPSS Inc, Chicago, Illinois, United States), and a $p$-value of less than 0.05 was considered to be statistically significant.

\section{Results}

The number of subjects treated via surgery and CRT were 91 and 96, respectively. The breakdown of subjects in the surgery and CRT groups is shown in - Table 1. The number of patients with recurrence in the surgery and CRT groups were $20(22.0 \%)$ and 25 (26.0\%), respectively. Three patients in whom clear residual tumors were recognized after CRT were excluded from the patients with recurrence in this study.

\section{Review on Time until Recurrence and Site of Recurrence}

Recurrence occurred within the first 2 years in 41 (91.1\%) of the 45 patients. The median interval until recurrence was 12 months, and the mean interval was $12.9 \pm 8.3$ months. The timing of recurrence in subjects in the surgery and CRT

Table 1 Breakdown of the patients included in this study

\begin{tabular}{|l|l|l|}
\hline & $\begin{array}{l}\text { Patients } \\
\text { treated with } \\
\text { surgery }\end{array}$ & $\begin{array}{l}\text { Patients } \\
\text { treated with } \\
\text { CRT }\end{array}$ \\
\hline Number of patients (total) & 91 & 96 \\
\hline $\begin{array}{l}\text { Follow-up period, median } \\
\text { (mo) }\end{array}$ & 37 & 40 \\
\hline $\begin{array}{l}\text { Number of patients with } \\
\text { recurrence }\end{array}$ & 20 & 25 \\
\hline Sex (M:F) & $14: 6$ & $18: 7$ \\
\hline Mean age (y) & $67.8(48-85)$ & $62.1(37-76)$ \\
\hline Primary location & \multicolumn{2}{|l|}{} \\
\hline Oral cavity & 8 & 0 \\
\hline Epipharynx & 0 & 5 \\
\hline Oropharynx & 1 & 7 \\
\hline Hypopharynx & 3 & 8 \\
\hline Larynx & 3 & 2 \\
\hline Nose/paranasal sinus & 2 & 2 \\
\hline Other & 3 & 1 \\
\hline Stage & 4 & 21 \\
\hline 1 & 4 & 0 \\
\hline 2 & 10 & 2 \\
\hline 3 & & \\
\hline 4 & 2 & 2 \\
\hline
\end{tabular}

Abbreviations: CRT, chemoradiotherapy; F, female; M, male. Notes: Oral cancer was common among patients with postsurgery recurrence, and oropharyngeal/hypopharyngeal cancers were more common in patients with post-CRT recurrence. There were many patients with advanced cancers among patients with post-CRT recurrences. groups has been shown separately (-Fig. 1). In the surgery and CRT groups, the mean interval until recurrence was $11.4 \pm 9.0$ months and $14.2 \pm 7.7$ months, respectively. No statistical significant difference was found between the two groups $(p=0.10)$.

The site of recurrence was local in 11 patients $(24.4 \%)$ and cervical lymph nodes in 17 (37.8\%), and there was occurrence of distant metastasis in 17 patients (37.8\%). The most frequent site of distant metastasis was the lung (11 patients), followed by the bone ( 4 patients), other ( 2 patients), intraabdominal lymph nodes ( 2 patients), and liver ( 1 patients). Simultaneous metastases were detected in the bone and intra-abdominal lymph nodes in one patient.

With respect to each treatment, recurrence was local or in cervical lymph nodes in approximately $60 \%$ of both the surgery and CRT groups. However, cervical recurrence was seen in 11 patients (55\%) in the surgery group, and there were more patients with local recurrence (9 patients) than with cervical recurrence (6 patients) in the CRT group (-Fig. 2).

\section{Review on Diagnostic Modalities that Led to Diagnosis of Recurrence}

The occasions that led to recurrence detection were reviewed mainly by dividing them into the following categories for analysis: (1) subjective symptoms; (2) findings at a regular examination (including endoscopy and palpation findings as well as neck ultrasound examination); and (3) imaging tests (performed as part of regular examination, such as CT and PET). Patients who complained of symptoms
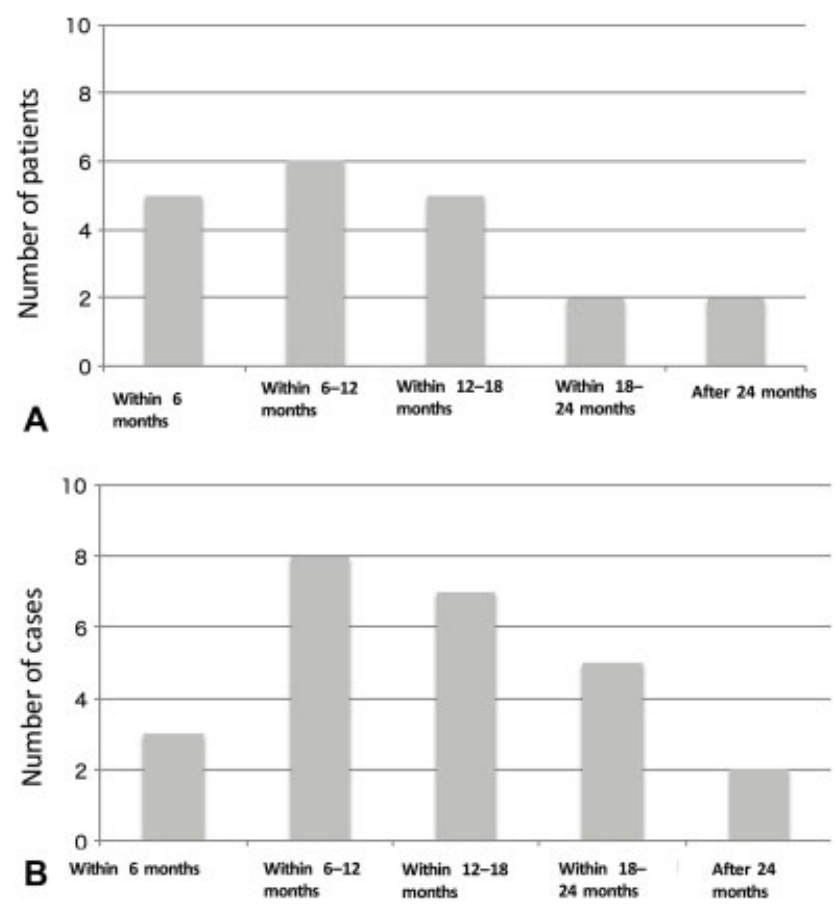

Fig. 1 Interval until recurrence. (A) Patients treated with surgery $(n=20)$; (B) patients treated with CRT $(n=25)$. A peak in the number of recurrences was observed at 6-12 months after treatment in both groups. CRT, chemoradiotherapy. 
Patients with surgery

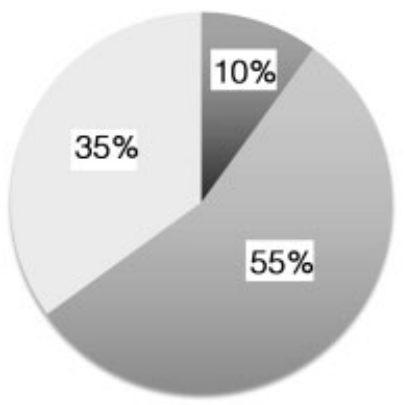

Patients with CRT

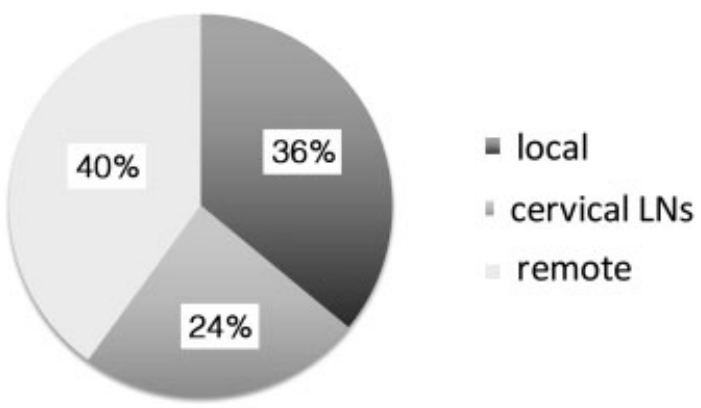

Fig. 2 The percentage of recurrence sites in patients treated with surgery and CRT. Neck recurrences were common among the patients treated with surgery, whereas there were slightly more local recurrences than those of neck recurrences in the patients treated with CRT. Remote metastasis was seen in $\sim 40 \%$ of both types of patients. CRT, chemoradiotherapy; LNs, lymph nodes.

at the time of regular examination were included in the subjective symptoms category.

Of all subjects with local recurrences, recurrences in $72.7 \%$ $(8 / 11)$ were detected by either (1) or (2); recurrence in cervical lymph nodes were detected at the same rate of $47 \%$; (1) + (2) versus (3); and all distant recurrences, $94.1 \%$ $(16 / 17)$, in subjects were detected by (3), except in one patient (-Fig. $\mathbf{3}$ ).

\section{Secondary Treatment and Prognosis after Diagnosis of Recurrence}

-Figs. 4, 5, and 6 present the results of our investigation of secondary treatment and prognosis after diagnosis of recurrence by recurrence site. Regarding prognosis, deaths in the cancer-bearing state were included in deaths by primary disease as a rule. However, the death of one subject owing to another type of cancer was considered as death from another cause.

Of the patients with local recurrence, salvage surgery was performed in five subjects (46\%) after detection of recurrence, and chemo/re-radiation therapy or best supportive

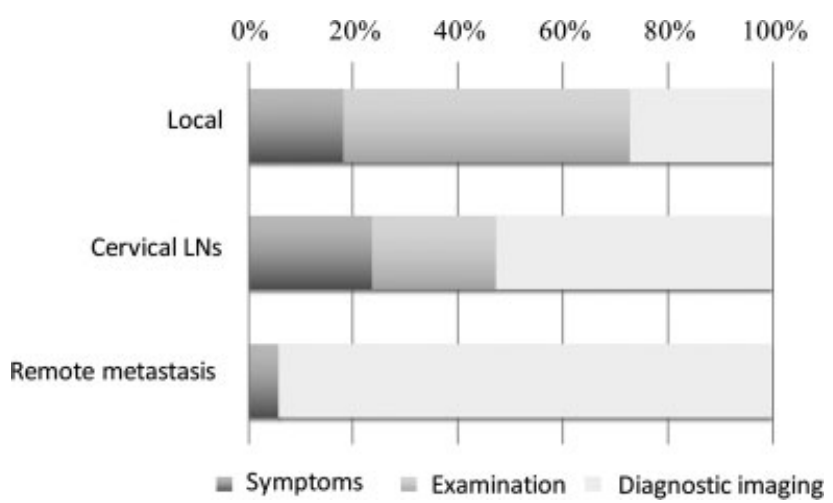

Fig. 3 Occasions that led to the diagnosis of recurrence. When examining based on recurrent site, local recurrence was often detected during a medical examination, whereas more than half of cervical lymph node metastases were detected by diagnostic imaging. Only one patient with remote metastasis was detected based on subjective symptoms, and the rest were identified with diagnostic imaging. LNs, lymph nodes. care was preferred in other patients owing to the difficulties or denial of surgery (-Fig. 4). With respect to subjects with cervical lymph node recurrence, cervical dissection was performed in 11 subjects (65\%), of which 6 (54.5\%) had no recurrence thereafter (-Fig. 5).

Chemotherapy was preferred for a majority of subjects with distant metastasis, except two, for whom surgery was performed. Both of these subjects had lung metastasis, and one exhibited no recurrence after surgery. Disease-free survival was not achieved in any subject who received chemo/re-radiation therapy (chemo/re-RT; - Fig. 6).

Finally, the results of the examination of the prognoses of subjects in whom recurrence occurred by a type of occasion that lead to diagnosis are shown in -Fig. 7. The median survival time was 2.5 months for the subjective symptom group, 27 months for the examination group, and 22 months for the imaging group. Thus, subjects in whom a subjective symptom led to the diagnosis had a poor prognosis (log-rank test, $p<0.01$ ).

\section{Discussion}

Although follow-up after treatment of patients with head and neck cancer aims for early detection and treatment of recurrence, there is no unified opinion on the follow-up regimen currently. This fact is also stated in the 2018 Head and Neck Cancer Clinical Practice Guidelines. ${ }^{1}$

The National Comprehensive Cancer Network (NCCN) guidelines state the following schedule as the follow-up recommendation: (1) medical examination: once every 1 to 3 months within 1 year after treatment, once every 2 to 6 months in the second year, and once every 4 to 8 months thereafter; (2) imaging: baseline imaging within 6 months after treatment; and (3) other: measurement of the level of the thyroid hormone every 6 to 12 months in subjects who have undergone neck irradiation and consideration of measurement of EBV DNA in subjects with nasopharyngeal cancer. $^{2}$ Currently, there is no mention of periodic imaging tests in these guidelines.

The follow-up observation regimen at our hospital is more thorough than that described in the above-mentioned 


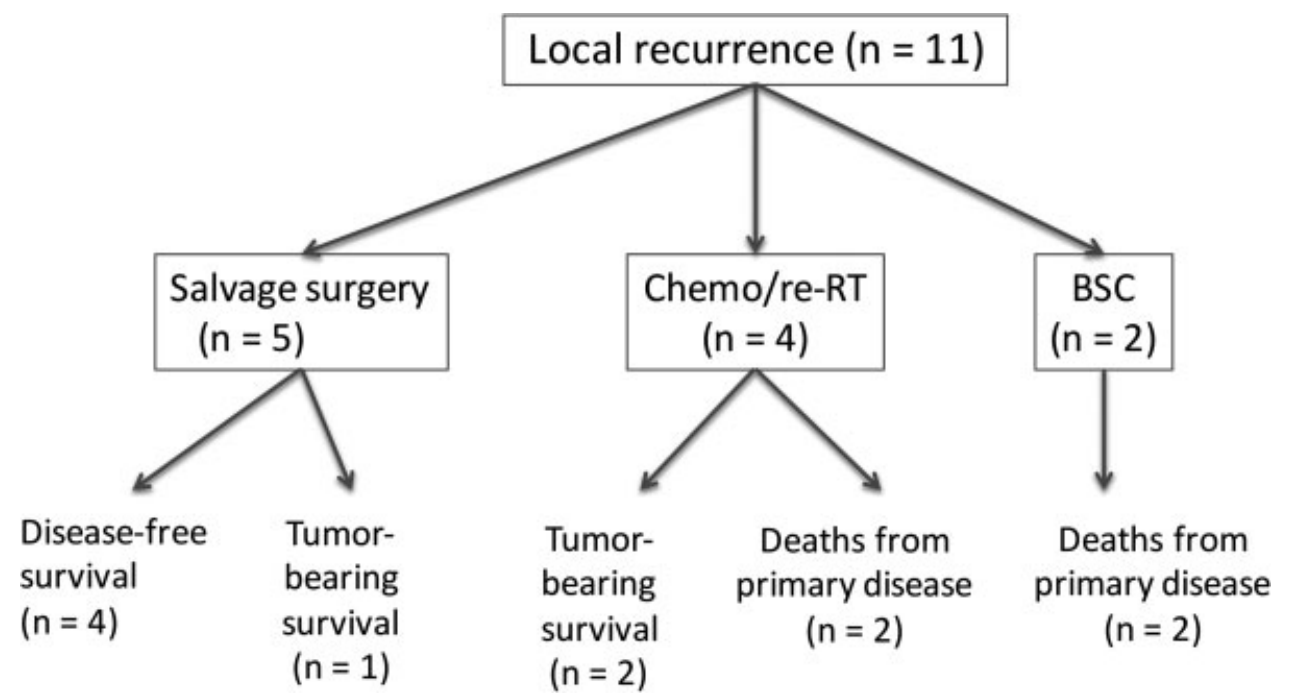

Fig. 4 Secondary treatment after diagnosis of local recurrence and its prognosis. Surgery was selected as the secondary treatment in $5 / 11$ (45.5\%) patients, prognosis for this group was relatively good; patients ultimately classified as disease-free survival accounted for $4 / 11$ ( $36.4 \%$ ). BSC, best supportive care.

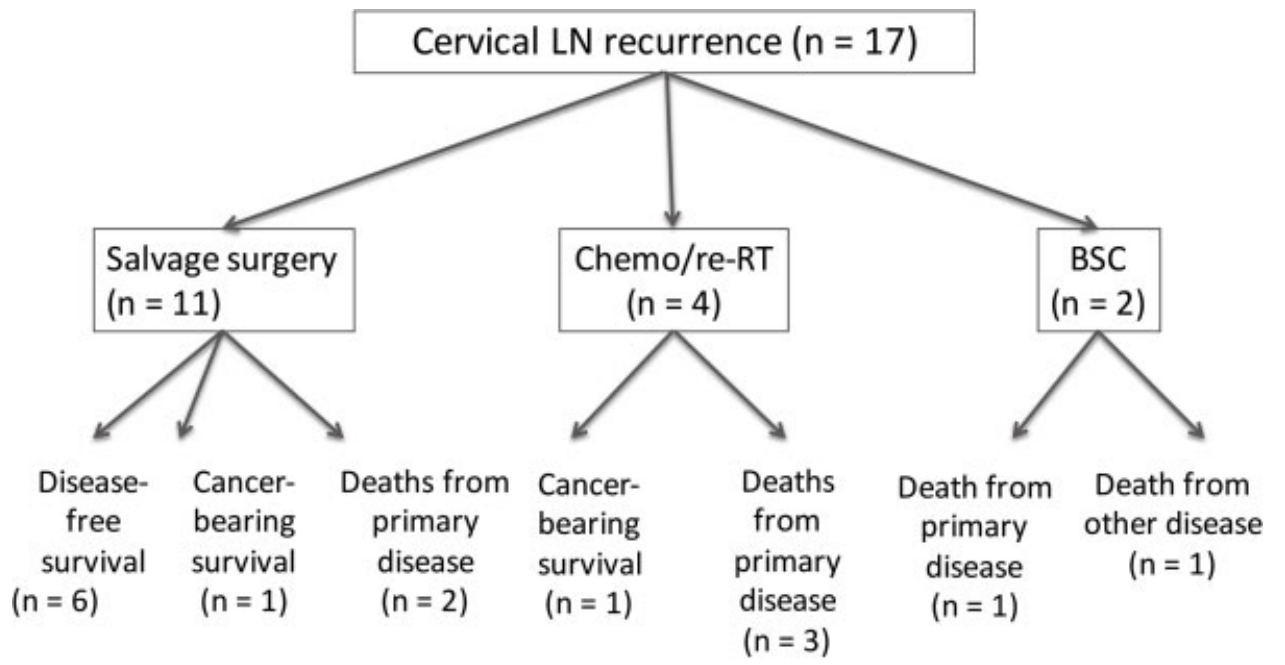

Fig. 5 Secondary treatment after cervical lymph node recurrence identification and prognosis. Surgery (neck dissection) was performed in 11/17 (64.7\%). Eventually, 5/11 (29.4\%) patients were salvageable. BSC, best supportive care; LN, lymph node.

guidelines in terms of the frequency of medical examination and, particularly, diagnostic imaging after baseline imaging. - Table 2 compares the Japanese and NCCN guidelines.

Although it has previously been reported that thorough follow-up observation did not improve the prognosis, ${ }^{3}$ high-level follow-up observation is permitted from the viewpoint of quality-adjusted life years. ${ }^{4}$ Thus, this is still debatable. Examination of subjects at our hospital indicated the possibility that recurrence diagnosis by medical examination and diagnostic imaging may contribute to improved prognosis. Therefore, although frequent examination and periodic imaging (including the initial imaging) may be useful, further investigation is required to determine whether such a thorough follow-up observation improves patients' quality of life. Our study also showed that blood collection and chest X-ray are not causes leading to recur- rence detection, and previous studies have not confirmed the utility of these tests either. ${ }^{5,6}$ Thus, the usage of these tests should be reduced/omitted.

Recurrence occurred within 2 years in $91.1 \%$ of patients, with the mean peak at approximately 12 months in our study. The results of the study conducted at another facility by Kissun et al also reported that in 49 out of 54 patients, recurrence occurred within 2 years after surgery, ${ }^{7}$ and this was similar to the results of the present study. Along with the NCCN guidelines, follow-up observation within the first 2 years was indicated to be important for diagnosis of recurrence.

Local/cervical lymph node recurrences accounted for approximately $60 \%$ in both surgery and CRT groups. Cervical lymph node recurrences were relatively frequently seen among the subjects who underwent surgery (11 patients). In 7 of these 11 patients, no cervical dissection was 


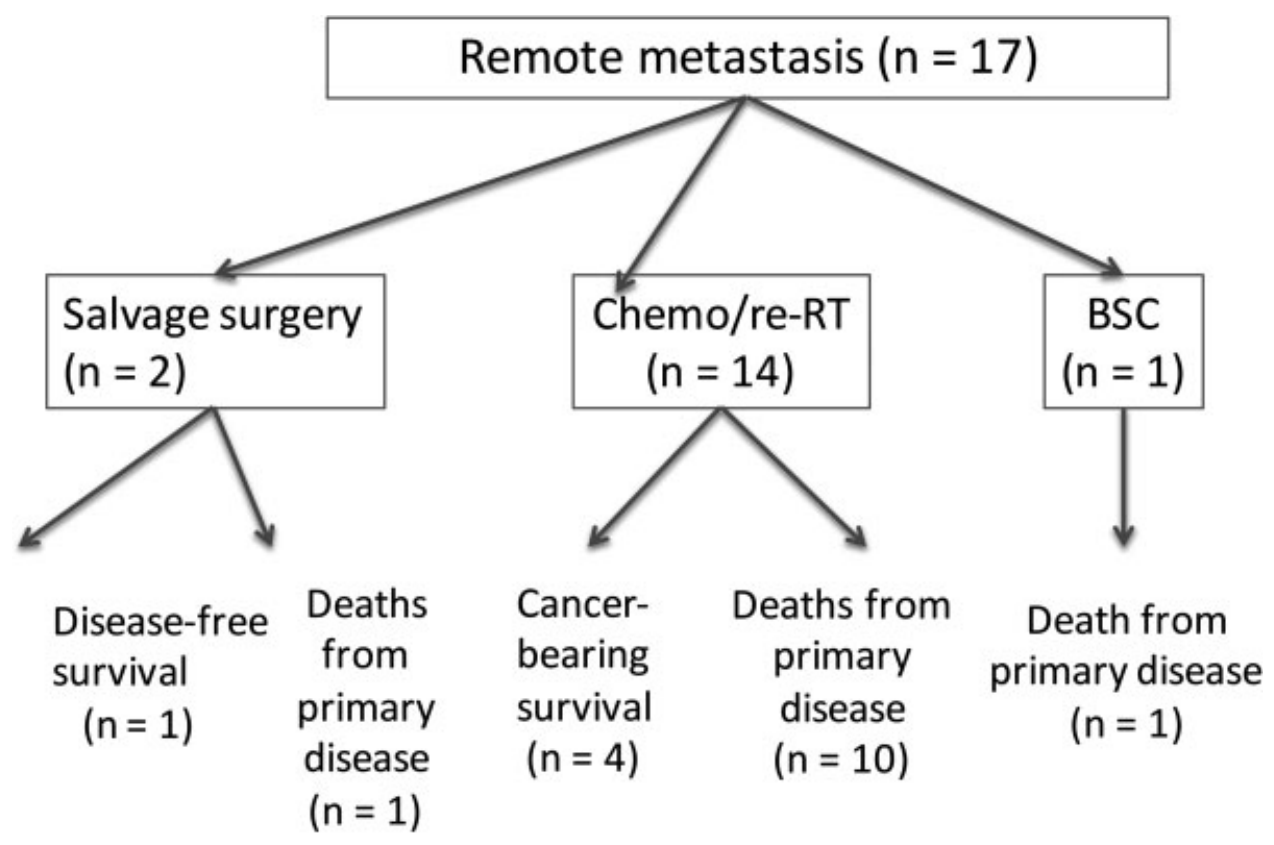

Fig. 6 Secondary treatment after cervical remote metastasis identification and its prognosis. Chemotherapy was preferred in majority of the patients. Surgery was performed for two patients in whom single lung metastasis was identified. One patient maintained disease-free status. BSC, best supportive care.

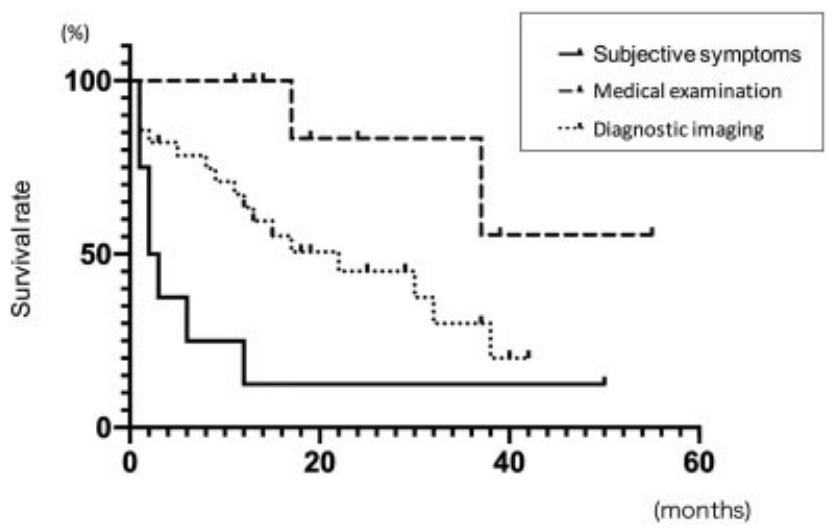

Fig. 7 Survival curve after diagnosis of recurrence according to the type of diagnostic modalities that led to diagnosis. When setting a censoring event as death owing to the primary disease, the median survival time for each group from when recurrence was diagnosed was 2.5 months for the subjective symptom group, 27 months for the examination group, and 22 months for the diagnostic imaging group. A significant difference was observed according to the log-rank test $(p<0.01)$.

performed as the initial treatment. Examination of these subjects showed that three subjects who underwent partial glossectomy and one with hypopharyngeal cancer who underwent treatment via endoscopic laryngopharyngeal surgery were included. With respect to tongue cancer, in particular, it has been reported that lymph node metastasis is present in 30 to $40 \%$ of subjects, even when the cancer is at stage I/II (clinically N0), ${ }^{8-10}$ and our study results indicated that careful attention to the diagnosis of cervical lymph node metastasis is necessary when partial glossectomy without neck dissection is chosen for the initial treatment.
In this study, remote metastasis was relatively common, and many of these subjects were diagnosed by conducting regular imaging tests. At our hospital, PET-CT has been actively performed with local evaluation by conducting CT and MRI in the past, which may have contributed to the fact that in 12 of the 16 subjects in whom remote metastasis was detected by imaging, metastasis was actually identified by PET. Except for lung metastasis, all remote metastases were identified by PET, indicating that PET is extremely useful in detecting remote metastasis.

PET has been reported to be useful in the diagnosis of recurrence $e^{11,12}$ and is also used in the assessment of objective response to CRT. The noninferiority of follow-up involving the usage of PET for conventional planned cervical dissection has been shown ${ }^{13}$; the use of PET in follow-up observation after treatment is important. Conversely, in the current medical care covered under health insurance, the use of PET for the diagnosis of recurrence is approved only when recurrence is suspected by conducting other imaging tests and only when this is observed in other patients. Therefore, for detecting remote metastasis, cervico-thoraco-abdominal CT appears to be a realistic policy at this moment.

Investigation of secondary treatment after diagnosis of recurrence and its prognosis showed that $4 / 11$ (36.4\%) subjects with local recurrence, $6 / 17$ (35.3\%) with neck recurrence, and $1 / 17$ (5.9\%) with remote metastasis recurrence were classified as having disease-free status. Because secondary treatment centering on surgery was conducted for almost all patients with disease-free survival, the main treatment for patients with head and neck cancer recurrence naturally appeared to be surgery. Therefore, it was considered important to diagnose local and cervical lymph node recurrence as early as possible while the metastatic lesions stay in conditions in which surgery is indicated. 
Table 2 Comparison of the Japanese Clinical Practice Guidelines for Head and Neck Cancer, the NCCN Guidelines, and the follow-up observation policy at our hospital

\begin{tabular}{|c|c|c|c|}
\hline & $\begin{array}{l}\text { Clinical Practice Guidelines } \\
\text { for Head and Neck Cancer } \\
\text { (2018 edition) }\end{array}$ & $\begin{array}{l}\text { NCCN Guidelines } \\
\text { (version 2. 2018) }\end{array}$ & $\begin{array}{l}\text { Follow-up observation } \\
\text { at our hospital }\end{array}$ \\
\hline $\begin{array}{l}\text { Medical examination } \\
\text { interval }\end{array}$ & No description & $\begin{array}{l}\text { - Year 1: Once every 1-3 mo } \\
\text { - Year 2: Once every 2-6 mo } \\
\text { - Year 3-5: Once every 4-8 mo }\end{array}$ & $\begin{array}{l}\text { - Year 2: every month } \\
\text { - Year 3-5: once every 2-3 mo }\end{array}$ \\
\hline Diagnostic imaging & $\begin{array}{l}\text { Baseline imaging (3-6 mo } \\
\text { after treatment) } \\
\text { - PET is useful for treatment } \\
\text { effect evaluation of CRT }\end{array}$ & $\begin{array}{l}\text { - Baseline imaging within } 6 \text { mo } \\
\text { after treatment } \\
\text { - Images should be taken when } \\
\text { symptoms/examination } \\
\text { findings suggestive of } \\
\text { recurrence are observed } \\
\text { - Chest CT with smoking } \\
\text { history } \\
\text { - Regular imaging if lesions are } \\
\text { in areas where direct } \\
\text { examination is difficult }\end{array}$ & $\begin{array}{l}\text { - Baseline imaging: 3-6 mo } \\
\text { after surgery } \\
\text { In the patients treated with CRT, } \\
\text { CT } 2 \text { mo and PET } 3 \text { mo after } \\
\text { treatment } \\
\text { - Imaging once } 6 \text { mo until the } \\
\text { end of year } 2 \text { after baseline } \\
\text { imaging (CT/MRI or PET) } \\
\text { - Once every } 6-12 \text { mo in year } \\
\text { 3-5 } \\
\text { - Chest X-ray: once every } 6 \text { mo }\end{array}$ \\
\hline Blood test & $\begin{array}{l}\text { - Tumor marker: Usefulness } \\
\text { not yet confirmed } \\
\text { - Check hypothyroidism }\end{array}$ & $\begin{array}{l}\text { - TSH: Once every 6-12 mo } \\
\text { - EBV DNA: only for } \\
\text { epipharyngeal cancer }\end{array}$ & $\begin{array}{l}\text { - Every } 6 \text { mo: blood count, } \\
\text { biochemistry, and tumor } \\
\text { markers }\end{array}$ \\
\hline
\end{tabular}

Abbreviations: CRT, chemoradiotherapy; CT, computed tomography; EBV DNA, Epstein-Barr virus DNA; MRI, magnetic resonance imaging; NCCN, the National Comprehensive Cancer Network; PET, positron emission tomography; TSH, thyroid-stimulating hormone.

Notes: There is no clear statement about the examination interval in the Clinical Practice Guidelines for Head and Neck Cancer. Only our follow-up observation policy indicates that regular imaging tests should be performed after baseline imaging.

Conversely, in reality, there are not many patients for whom surgery can be chosen as the secondary treatment. Therefore, from this point of view, the survival time after diagnosis of recurrence and evaluation of the quality of survival also appear to be important factors. In the patients included in our study, the results of the investigation of survival time from the diagnosis of recurrence, which was conducted using the Kaplan-Meier method by considering death resulting from the primary disease as a censoring event, showed that there was no significant difference between the surgery and chemo/re-RT groups in terms of secondary treatment (log-rank test; $p=0.77)$ and that the median survival time was longer in the chemo/re-RT group than in the surgery group (surgery group: 22 months; chemo/re-RT: 30 months; -Fig. 8). This result may be influenced by the fact that the chemo/re-RT group includes cancer-bearing survival to some extent and the follow-up period may be too short to evaluate the survival time of the different type of secondary treatment.

The analysis of the survival period based on the type of occasion that led to recurrence diagnosis also showed that the prognosis of the patients, in whom subjective symptoms led to diagnoses of recurrence, was significantly worse than that of those in which medical examination or diagnostic imaging led to diagnosis of recurrence ( $\boldsymbol{- F i g . ~} \mathbf{7}$ ). In such analysis, the lead time bias, which is an apparent extended survival period owing to early detection, cannot be completely excluded. However, $60 \%$ (6/10) of patients with recurrence detected during medical examination remained disease free, whereas only $14 \%$ of those in whom recurrence was detected by subjective symptoms reached the disease-free survival state

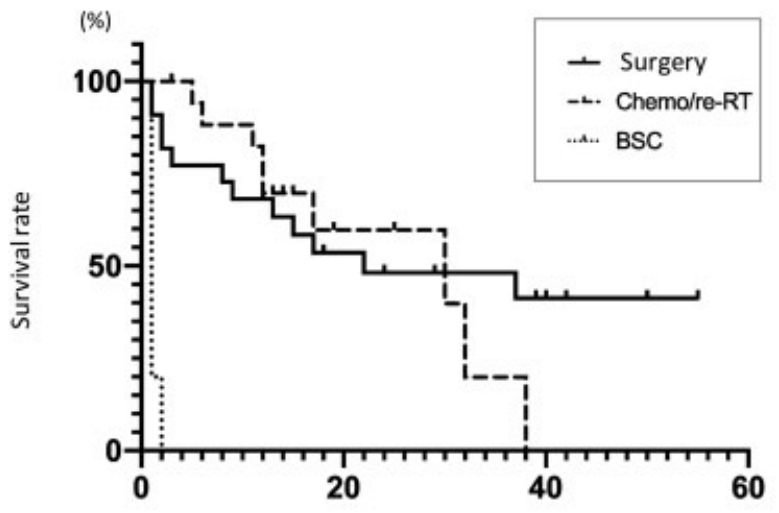

(months)

Fig. 8 Survival curve for each secondary treatment modality after diagnosis of recurrence. When censoring on death from primary disease, no significant difference was found in the log-rank test when the surgery and chemo/re-RT treatment groups were compared. Ope, surgery; Chemo/re-RT, chemotherapy or re-radiation therapy; BSC, best supportive care.

after secondary treatment, and this indicated a large difference between the two. This indicated that detection during a medical examination may contribute to improved prognosis. In recent years, new drugs, such as nivolumab, ${ }^{14,15}$ have enabled the extension of patient survival. Thus, it will become even more important in the future to detect recurrence of the condition in which the quality of life before appearance of subjective symptoms can be maintained.

The real purpose of follow-up observation may be to improve life prognosis, including salvage and quality of 
recurrence cases. Based on this study, issues that need to be investigated in the future include the following: (1) thorough follow-up observation focused on patients susceptible to recurrence (in particular, those patients for whom salvage is either necessary or possible); (2) investigation of treatment methods and prognosis of the patients in whom recurrence is believed to be able to be detected early during examination or with the usage of diagnostic imaging techniques; (3) quality of life during the survival period and analysis, including the economic point of view. We believe that a prospective comparative study on the earlier-mentioned items will lead to a truly appropriate follow-up observation regimen.

\section{Summary}

We investigated patients in whom surgery or chemoradiotherapy was performed as the initial treatment and recurrence occurred after that. Because recurrence occurred in $91.1 \%$ of the patients within the first 2 years, this period was considered to be particularly important. Of the subjects who underwent surgery, particular attention should be paid to subjects with cervical lymph node metastasis of oral cancer, in whom neck dissection was not performed. Many distant metastases were detected via diagnostic imaging, especially by PET, indicating the usefulness of PET for the detection of metastasis even before the symptoms appear.

In the secondary treatment for recurrence, the main approach used currently that aims at achieving diseasefree survival is the surgical treatment. The diagnosis of recurrence at a stage when surgery is possible is particularly important for patients in whom local or cervical lymph node recurrences are involved. In the analysis of prognosis based on the occasion, prognosis of patients in whom recurrence was diagnosed during an examination or by imaging was better than that of patients in whom subjective symptoms led to diagnosis, which was indicative of the possibility that prognosis can be improved by implementing thorough follow-up observations.

Conflict of Interest

None declared.

\section{References}

12018 Japanese Clinical Practice Guidelines for Head and Neck Cancer (Japanese Society for Head and Neck Cancer edition). Tokyo: Kanehara \& Co. Ltd.; 2018:85

2 National Comprehensive Cancer Network. NCCN Clinical Practice Guidelines in Oncology: Head and Neck Cancers; 2018

3 Schwartz DL, Barker J Jr, Chansky K, et al. Postradiotherapy surveillance practice for head and neck squamous cell carcinoma-too much for too little? Head Neck 2003;25(12):990-999

4 Meregaglia M, Cairns J, Licitra L, Bossi P. The use of intensive radiological assessments in routine surveillance after treatment for head and neck cancer: an economic evaluation. Eur J Cancer 2018;93:89-98

5 Guerra EN, Rêgo DF, Elias ST, et al. Diagnostic accuracy of serum biomarkers for head and neck cancer: a systematic review and meta-analysis. Crit Rev Oncol Hematol 2016;101:93-118

6 O'Meara WP, Thiringer JK, Johnstone PA. Follow-up of head and neck cancer patients post-radiotherapy. Radiother Oncol 2003;66 (03):323-326

7 Kissun D, Magennis P, Lowe D, Brown JS, Vaughan ED, Rogers SN. Timing and presentation of recurrent oral and oropharyngeal squamous cell carcinoma and awareness in the outpatient clinic. Br J Oral Maxillofac Surg 2006;44(05):371-376

8 D'Cruz AK, Vaish R, Kapre N, et al; Head and Neck Disease Management Group. Elective versus therapeutic neck dissection in nodenegative oral cancer. N Engl J Med 2015;373(06):521-529

9 Asakage T. Neck treatment in early tongue cancer. Oto-RhinoLaryngology Tokyo 2017;60:218-225

10 Imai T, Satoh I, Matsumoto K, et al. Retrospective observational study of occult cervical lymph-node metastasis in T1N0 tongue cancer. Jpn J Clin Oncol 2017;47(02):130-136

11 Yoo J, Henderson S, Walker-Dilks C. Evidence-based guideline recommendations on the use of positron emission tomography imaging in head and neck cancer. Clin Oncol (R Coll Radiol) 2013; 25(04):e33-e66

12 Fletcher JW, Djulbegovic B, Soares HP, et al. Recommendations on the use of 18F-FDG PET in oncology. J Nucl Med 2008;49(03): 480-508

13 Mehanna H, Wong WL, McConkey CC, et al; PET-NECK Trial Management Group. PET-CT Surveillance versus neck dissection in advanced head and neck cancer. N Engl J Med 2016;374(15): 1444-1454

14 Ferris RL, Blumenschein G Jr, Fayette J, et al. Nivolumab for recurrent squamous-cell carcinoma of the head and neck. $\mathrm{N}$ Engl J Med 2016;375(19):1856-1867

15 Ferris RL, Blumenschein G Jr, Fayette J, et al. Nivolumab vs investigator's choice in recurrent or metastatic squamous cell carcinoma of the head and neck: 2-year long-term survival update of CheckMate 141 with analyses by tumor PD-L1 expression. Oral Oncol 2018;81:45-51 